\title{
A study on modeling of a piezoelectric motor
}

\author{
Youssef Baba ${ }^{1}$, Mostafa Bouzi \\ ${ }^{1}$ University Hassan II, ENSAM, 20670 Casablanca, Morocco \\ ${ }^{2}$ University Hassan I, FST, 26000 Settat, Morocco
}

\begin{tabular}{l}
\hline \hline Article Info \\
\hline Article history: \\
Received Jun 27, 2020 \\
Revised Mar 1, 2021 \\
Accepted Mar 21, 2021 \\
\hline
\end{tabular}

Keywords:

Fuzzy logic

Modeling

Temperature effect

Ultrasonic motor

USR60

\begin{abstract}
In this paper, a study on modeling of a piezoelectric transducer type rotary traveling wave ultrasonic motor (USM) is presented. First a mathematical model and numerical simulation results are achieved. The model is based on the theory of piezoelectricity and physic theory. An experimental model is worked out and compared to the numerical model. The influence of the temperature on characteristics such as the rotational speed of the motor is considered. The speed of the USM is measured at temperature between $17^{\circ} \mathrm{C}$ and $50^{\circ} \mathrm{C}$. To develop suitable control strategies for the drive, a fuzzy model type Takagi-Sugeno is used. The unknown parameters of the output membership functions are determined by least square method. Experimental data are used to examine the validity of the fuzzy model. Comparison between experimental and calculated data of the fuzzy model indicates that the fuzzy model can well describe the nonlinear characteristics among the frequency of driving voltage and rotating speed.
\end{abstract}

This is an open access article under the CC BY-SA license.

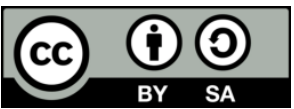

Corresponding Author:

Youssef Baba

University Hassan II

ENSAM, 20670 Casablanca Morocco

Email: youssef.baba@gmail.com

\section{INTRODUCTION}

Ultrasonic motors are a type of actuator which have some excellent performances such as high holding torque, high torque at low speed, quiet operation, simple structure, compact size and no electromagnetic interference [1].

Due to such characteristics, ultrasonic motors have been in many practical applications, such as in robots, medical instruments, cameras, aeronautics, MEMS and many others. Precise theoretical modeling of motion and energy conversion in ultrasonic motors is of great interest both from theoretical and practical viewpoints. One of the most interesting types of motors is a travelling wave ultrasonic motor. However, it is difficult to derive complex mathematical model of the USM. Moreover, the control characteristics of the motor are highly nonlinear.

The exact values of motor parameters are difficult to obtain and they are time-varying due to increase in temperature and changes in motor drive operating conditions such as driving frequency, and load torque. Several attempts have been carried out to develop the model of the motor. The equivalent circuit was widely used on ultrasonic motor modeling [1]-[6]. But this type of model is a rude modeling method of the dynamic characteristics of ultrasonic motor and cannot be used to design motor's controller directly. Dongsheng Zhang, Shiyu Wang and Jie Xiud [7] developed an analytical dynamic model of ring ultrasonic motor. Based on this model, the piezoelectric parametric effects on the wave distortion and contact mechanics are examined. In [8]-[10] differential evolution algorithm is used for motor's model identification. The algorithm reduces the time required in the process of identification. Many researchers considered mechanical characteristic of contact frictional layer and calculated displacement and speed of the rotor using 
finite element method [11]-[13]. In [14]-[16] dynamic contact problems between a rigid rotor and deformable stator were approximately solved. Due to the limitations of theoretical modeling, control design usually adopts identification method based on the tested data. According to the different identification methods, the forms of model can be the transfer function, differential equation, neural network, and so forth. In recent years, fuzzy modeling method based on fuzzy reasoning is gradually arisen. The same as in the neural network model, fuzzy model is also based on experimental data, easy to show the nonlinear information. The fuzzy method is mostly used to realize speed and position control [17]-[19].

This paper presents both a numerical model and a fuzzy modeling method based on Takagi-Sugeno reasoning is used to obtain the model of ultrasonic motor. The Takagi-Sugeno model is chosen to make the proposed model easy to use on control design. The output of Takagi-Sugeno model is a linear mathematical expression. Contrariwise, the Mamdani model gives a fuzzy output. The proposed work considers the effect of temperature. This work will be very useful for the optimal design, reducing the heat loss, preserving the desired rotary speed, and improvement of precise control of USM. This method is based on fuzzy model structure and experimental measurements. The data contains the driving frequency, the temperature and rotary speed of ultrasonic motor. The least squares method is used to identify the unknown parameters of the conclusions of fuzzy rules.

This paper is organized as in Section 2, a mathematical model is presented. Simulations are achieved on MATLAB. In Section 3, experiments are performed to verify the effectiveness of the analytical model. The effect of temperature is discussed. An adaptive fuzzy model is investigated in Section 4, taking into account the motor's non-linearity. Finally, conclusions of this work are presented in Section 5.

Table 1. Design specifications and simulation parameters of USR60

\begin{tabular}{lll}
\hline Items & Descriptions & Values \\
\hline$f$ & Supply frequency & $40-42 \mathrm{kHz}$ \\
$u$ & Supply voltage & $100 \mathrm{~V}_{\mathrm{rms}}$ \\
Speed & Rated speed & $90 \mathrm{rpm}$ \\
Power & Rated power & $3 \mathrm{w}$ \\
$M_{L}$ & Load torque & $0-0.32 \mathrm{Nm}$ \\
$M$ & Stator mass & $10.1 \mathrm{e}-3 \mathrm{Kg}$ \\
$\mathrm{m}_{\mathrm{R}}$ & Rotor mass & $30 \mathrm{e}-3 \mathrm{Kg}$ \\
$d_{Z}, d_{R}$ & Rotor damping & $5 \%$ \\
$D$ & Stator damping & $15.4 \mathrm{Ns} / \mathrm{m}$ \\
$C$ & Stiffness & $5.95 \mathrm{e}+8 \mathrm{~N} / \mathrm{m}$ \\
$F_{N}$ & Applied force & $160 \mathrm{~N}$ \\
$J$ & Moment of inertia of the rotor & $7.2 \mathrm{e}-6 \mathrm{Kgm}$ \\
$\Theta$ & Coupling factor & $0.2263 \mathrm{~N} / \mathrm{V}$ \\
\hline
\end{tabular}

\section{USM MODELING}

In [20] an equivalent mechanical two-mode approximation is given, representing the modal amplitudes $w_{1, t}$ and $w_{2, t}$ of the sine- and cosine-mode of the vibrating system (stator and ceramic), see Figure 1. The parameter $m$ describes the modal mass of stator and ceramic, and $d_{S}$ represents the structural damping. $c_{S}$ and $c_{C}$ reflect the equivalent mechanical stiffness of the stator and ceramic, respectively. $y_{1}$ and $y_{2}$ are displacements which are proportional to the electric currents flowing into the ceramic layer. Crosscoupling between the two modes is represented by the small disturbances $\varepsilon_{1}, \varepsilon_{2}$. The determination of the model parameters can be based on energy considerations or detailed finite element analysis of the stator [21][24].

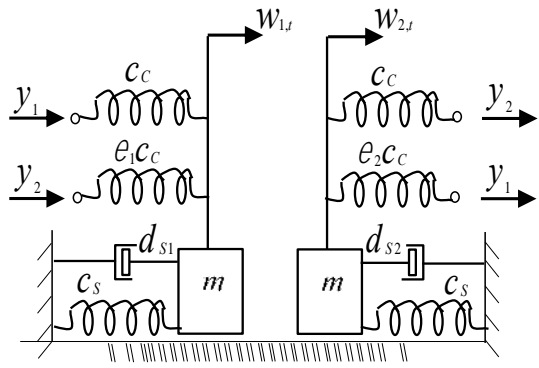

Figure 1. Mechanical model for the (stator + ceramic) of USM 


\subsection{Mathematical model}

An ultrasonic motor uses mechanical vibrations in the ultrasonic range as its drive source. A voltage is applied to the piezoelectric ceramic elements through two electrodes to generate alternating expansions and contractions in the ceramic body. The magnitude of these oscillations is of the order of $1 \mu \mathrm{m}$. In order to obtain a higher gain; the resonance effect of the ceramics in the ultrasonic range is used. The specifications of the USR60-object of this study-are given in Table 1. The stator of USM is considered as a piezoelectric plate, polarized in its thickness direction [1].

When two specific phase voltages are applied to the piezoelectric elements, a travelling wave is generated on the surface of the stator. The travelling wave produces vibration with an elliptic locus on the surface of the stator. This stator vibration is transformed into a rotation through friction contact between the stator and rotor. The rotor is pressed against the stator by a disc spring $(160 \mathrm{~N})$. The contact area between stator and rotor moves with the travelling wave. The rotor is modeled by a rigid strip [1]. In dealing with the dynamics of the rotor, two degrees of freedom must be taken into account: first the rotation of the rotor and second the motion in $z$ direction, as shown by [25]. The dynamics of the vertical rotor motion is obtained through the force equilibrium in $z$ direction.

$$
m_{R} \ddot{z}+d_{Z} \dot{z}=F_{Z}-F_{N}
$$

with $m_{R}$ is the mass of the rotor, and $d_{Z}$ is an appropriate damping term of the vertical motion. The applied axial load, $F_{N}$, is a system input, and $F_{Z}$ is the force at the contact area. The equation of rotational motion is calculated by.

$$
J \ddot{\theta}+d_{R} \dot{\theta}=M_{R}-M_{L}
$$

where $M_{L}$ is the applied torque (mechanical load), $d_{R}$ denotes the damping in spinning direction and $J$ represents the moment of inertia of the rotor. The $M_{R}$ term is the frictional pressure at the contact area. The $d_{R}$ term is small, but not negligible. The dynamics of the stator can be described by

$$
M \cdot \ddot{w}_{t}+D \cdot \dot{w}_{t}+C \cdot w_{t}=\Theta \cdot u
$$

where the parameter $M$ describes the model mass vector of stator and ceramic, $D$ reflects the structural damping vector, and $C$ represents the equivalent mechanical stiffness vector of the stator and ceramic. The vector $u$ is the phase voltages, and $\Theta$ represents the electromechanical coupling matrix considering the cross excitation. In order to consider the interaction between the stator/rotor-contact, Equation (3) reflecting the stator's dynamics is to extend by a nonlinear modal force vector $F_{d}$ yielding.

$$
M \cdot \ddot{w}_{t}+D \cdot \dot{w}_{t}+C \cdot w_{t}=\Theta \cdot u+F_{d}
$$

Hence, the dynamics of the motor is completed. An integration of this modeling approach based on closed form solutions for the contact mechanism into the simulation model is feasible.

\subsection{Simulation results}

The implementation is performed on MATLAB and the parameters of USM are scheduled in Table 1. The temperature of the motor is supposed to be $20^{\circ} \mathrm{C}$. The speed of the motor has its maximum at the mechanical resonant frequency. It is due to the fact that the revolving speed of the motor is proportional to the vibration force of piezoelectric elements. So, any deviation from this frequency degrades the motor performance. However, this effect seems more serious for frequency decrements.

To avoid these phenomena, speed of USM was controlled in 40-42kHz frequency range. Figure 2 shows speed-frequency characteristic of USM under different load torques. Speed-frequency characteristic of USM is not linear. Rotary speed decreases as the load torque increases. As mentioned, variations in driving frequency changes speed of USM. Figure 3 represents speed-torque characteristic of USM for driving frequency $f=40 \mathrm{kHz}$. When the load torque exceeds $0.32 \mathrm{Nm}$, the model becomes imprecise. 


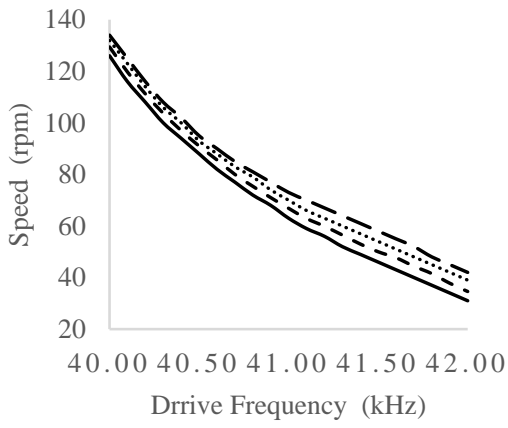

Figure 2. Speed-frequency characteristics of USM

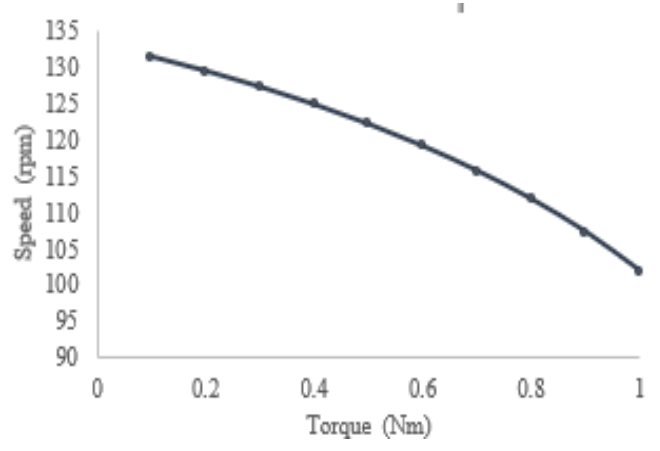

Figure 3. Speed-torque characteristic USM

\section{EXPERIMENTAL DATA AND RESULTS}

To obtain the temperature and the performance characteristics of the motor, an experiment setup is established. The motor is alimented by the two-phase voltages with $90^{\circ}$ phase shift. The teensy development board running at $72 \mathrm{MHz}$ and the driver circuit are utilized to generate, amplifier, and filter the applied voltages of the USM.

A temperature sensor (LM35) is used to measure the temperature of the stator in the temperature range of $17^{\circ} \mathrm{C}$ to $50^{\circ} \mathrm{C}$. A highest speed of the USM can be obtained when the stator is excited by the resonant frequency. The driving frequency is measured by oscilloscope. A capacitive encoder (AMT102 with 1024 pulses per revolution) is used to measure the rotating speed of USM. The development board is used to measure data from both the position and temperature sensors. The experimental bench and its block diagram are shown in Figure 4.

A comparison between simulation and experimental results for speed-frequency characteristic of USM under different load torques, is given in Figure 5. The figure shows that the simulation results are compatible with the experimental values. However, for frequencies above $41.1 \mathrm{kHz}$ we notice a slight deviation due to the increase in motor temperature. The matlab model is not time dependent. Nevertheless, during motor operation and as time passes, the temperature of the ceramic elements increases, which decreases the USM speed.

In order to measure the temperature of the USM, the stator is excited by the resonant frequency. Figure 6 shows the speed of USM versus time. It is evident that speed decreases according to the time dependent temperature rises. Figure 7 shows the measured temperature versus time. After 13 minutes, the motor temperature exceeds $50^{\circ} \mathrm{C}$. The temperature-time curve is almost linear. In terms of its effect on resonant frequency, however, temperature change is a more important factor than dimensional precision. The effect of temperature is shown in Figure 8. From this figure, it is concluded that the resonant frequencytemperature characteristic is linear from $20^{\circ} \mathrm{C}$ to $50^{\circ} \mathrm{C}$, and it can be approximated by a linear equation [26][27]. In [1] the authors affirmed that this characteristic is linear from $-10^{\circ} \mathrm{C}$ to $55^{\circ} \mathrm{C}$.
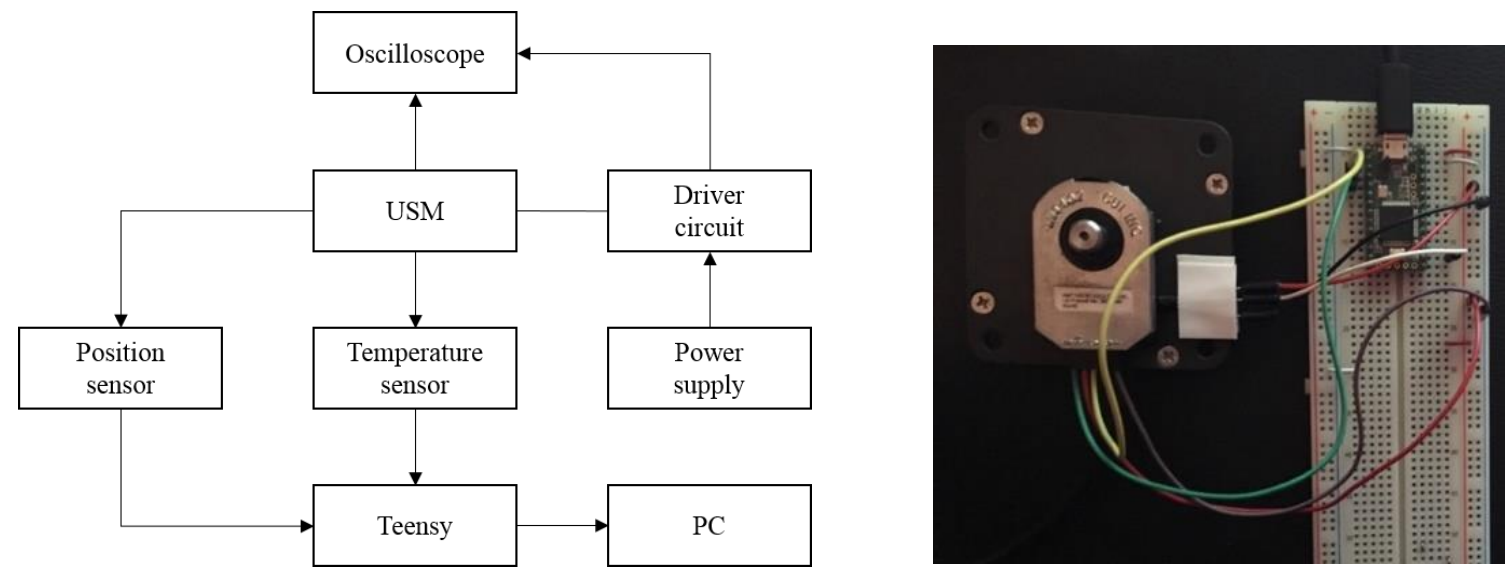

Figure 4. Experimental bench and its block diagram 

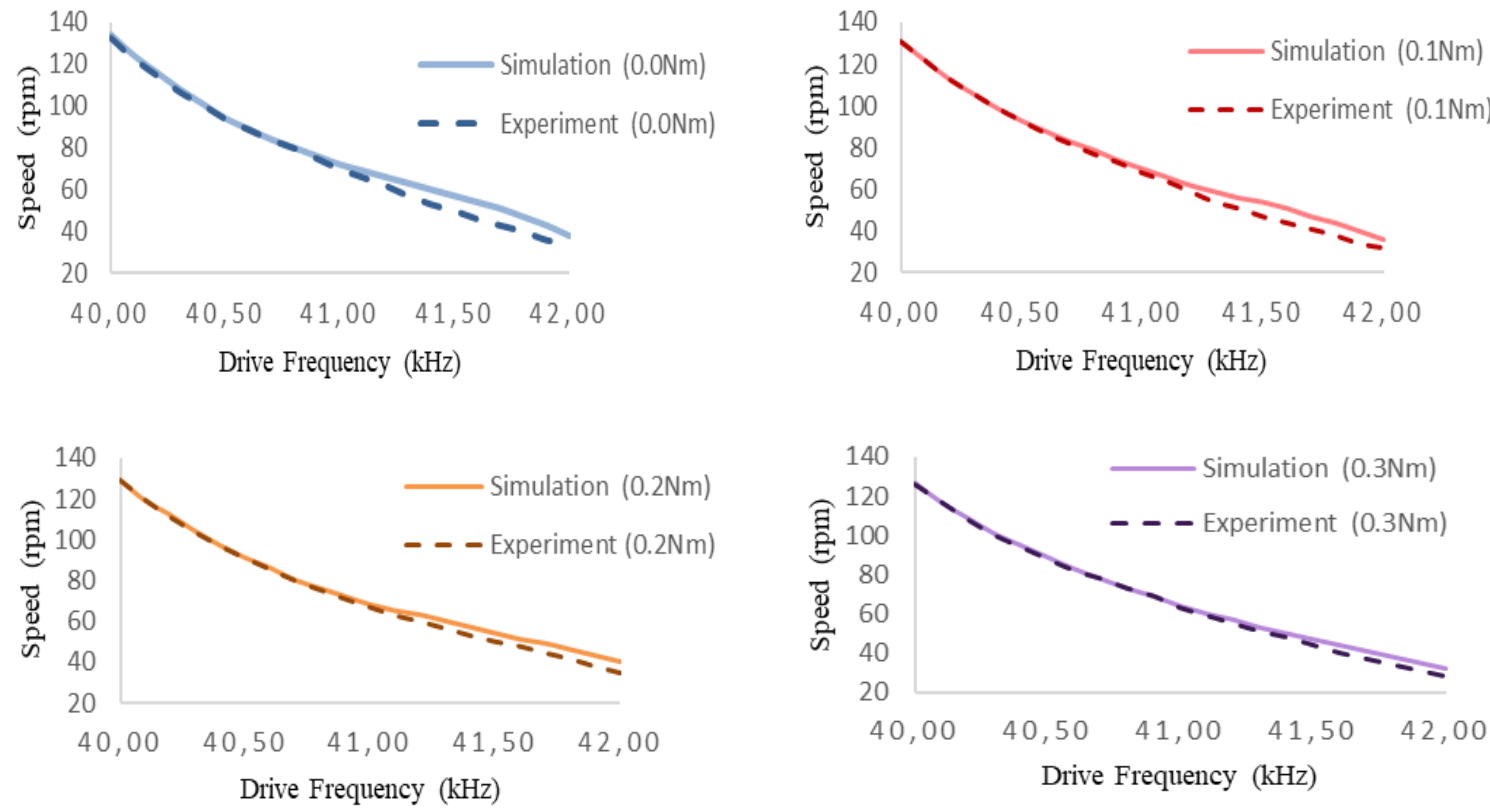

Figure 5. Comparison between simulation and experiment results

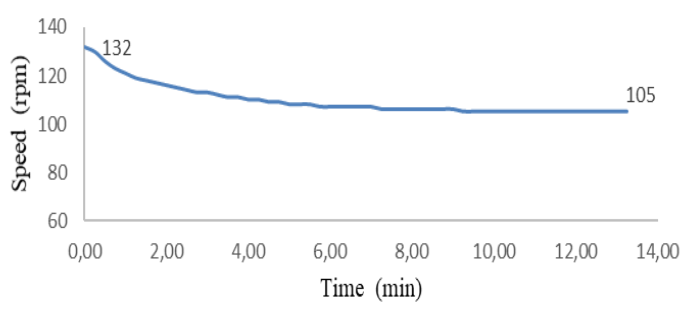

Figure 6. Speed-time characteristic of USM

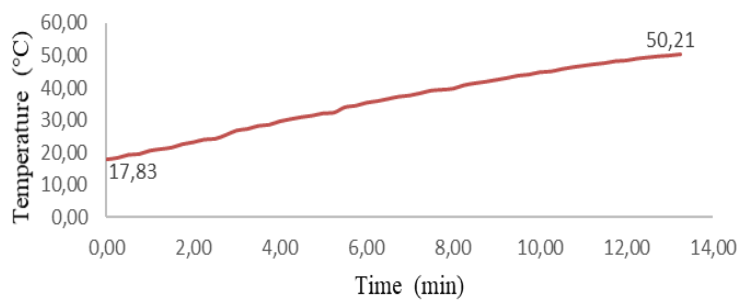

Figure 7. Temperature of the USM versus time

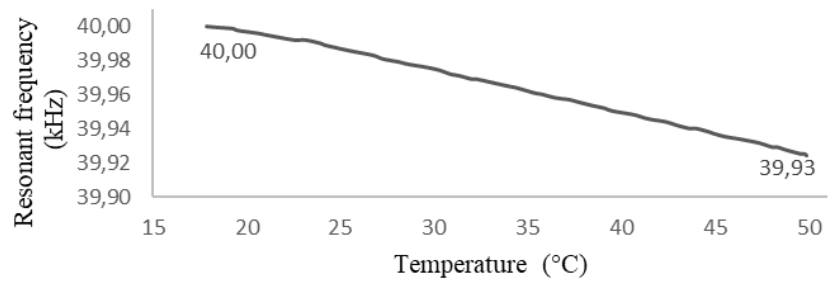

Figure 8. Temperature-dependence of resonant frequency

\section{ADAPTIVE FUZZY MODEL}

The ultrasonic motor has nonlinear speed characteristics which vary with drive operating conditions such as temperature rise and applied load torque changes. Furthermore, the motor has no mathematic model for control by far because the drive principle is different from the general electromagnetic type motors. In this section, a fuzzy model is proposed to solve these problems from the point of utility. The input of the fuzzy model is the frequency $f$, and the output is the speed $\omega$. The frequency $f$ is defined in the range from 40 to $42 \mathrm{kHz}$. Looking at the input-output data shown in Figure 5, we can see that the speed-frequency characteristics change as the frequency decreases. Five fuzzy sets: "very_small", "small", "medium", "big", and "very_big" are defined as shown in Figure 9. We have a model with the five implications.

$$
\text { if } f \text { is very_small then } \omega_{1}=a_{1} \cdot f+b_{1}
$$


if $f$ is small then $\omega_{2}=a_{2} \cdot f+b_{2}$

if $f$ is medium then $\omega_{3}=a_{3} \cdot f+b_{3}$

if $f$ is big then $\omega_{4}=a_{4} \cdot f+b_{4}$

if $f$ is very_big then $\omega_{5}=a_{5} \cdot f+b_{5}$

where $\omega_{i}(\mathrm{i}=1 \ldots 5)$ is the rotary speed. The five membership functions of Figure 9 are given by.

$$
\begin{aligned}
& m_{1}(f)=\exp \left(-\sigma(f-40000)^{2}\right) \\
& m_{2}(f)=\exp \left(-\sigma(f-40500)^{2}\right) \\
& m_{3}(f)=\exp \left(-\sigma(f-41000)^{2}\right) \\
& m_{4}(f)=\exp \left(-\sigma(f-41500)^{2}\right) \\
& m_{5}(f)=\exp \left(-\sigma(f-42000)^{2}\right)
\end{aligned}
$$

with $\sigma=6,65 e^{-6}$. Then the motor speed is obtained by defuzzification.

$$
\omega=\sum_{1}^{5} \beta_{i} \cdot \omega_{i}=P^{T} \psi
$$

with $\beta_{i}=m_{i} / \sum_{1}^{5} m_{i}, P^{T}=[a, b]$, and $\psi=[f, 1]^{T}$. In this paper the vector $P$ is calculated by the least squares algorithm.

$$
\begin{aligned}
& P_{i+1}=P_{i}+S_{i+1} \psi_{i+1}\left(\omega_{i+1}-\psi_{i+1}^{T} P_{i}\right) \\
& S_{i+1}=S_{i}-\frac{S_{i} \psi_{i+1} \psi_{i+1}^{T} S_{i}}{1+\psi_{i+1}^{T} S_{i} \psi_{i+1}} \\
& i=1 \ldots 999 \\
& P_{0}=0, S_{0}=1000 \cdot I
\end{aligned}
$$

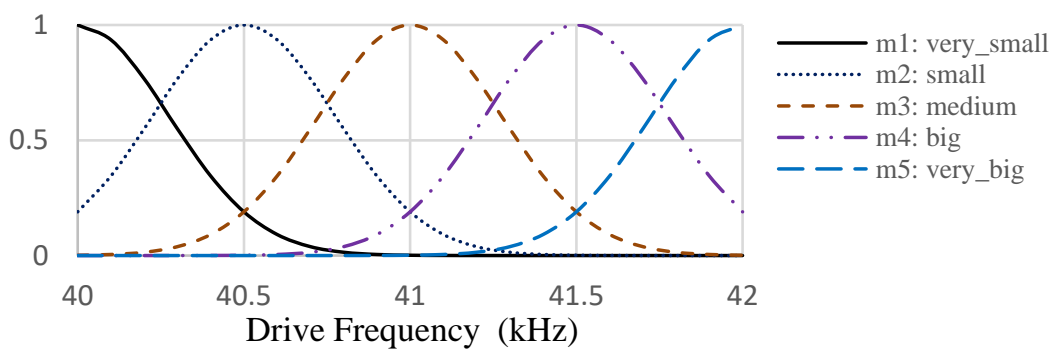

Figure 9. Membership functions of fuzzy sets

Figure 10 shows the experimental model (no load) and the fuzzy model of the speed-frequency characteristic. Such characteristic indicates the similarities between the experimental nonlinear model and the fuzzy model. This model can then be used to perform a direct control design. With the adaptive fuzzy approach, motor's nonlinearities are masked by local linearization using the Takagi-Sugeno reasoning. The same conclusions are valuable for load below $0.3 \mathrm{Nm}$. 


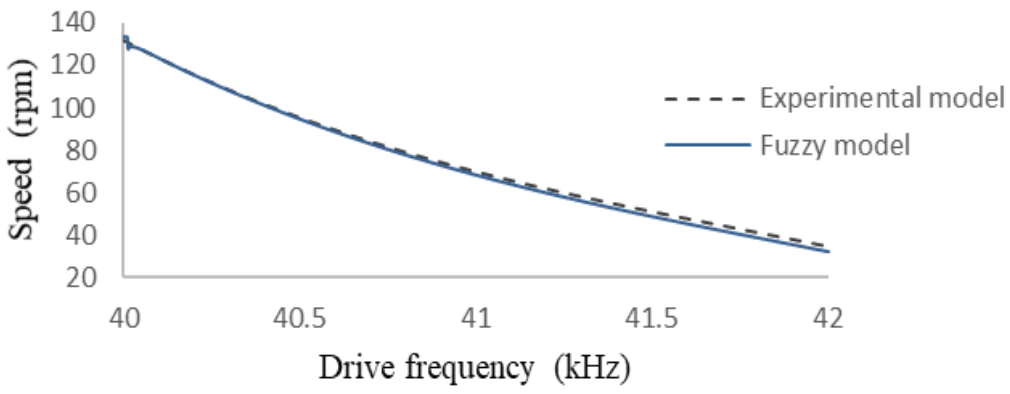

Figure 10. The speed-frequency characteristic

\section{CONCLUSION}

In this paper, a study on modeling of USM is presented. A numerical model of the motor is achieved. The simulation results are compared with the experimental ones which give good agreement of the model. The effect of the temperature on the characteristics of the USM is discussed. Furthermore, the effects of driving frequency and load torque are identified. Based on the experimental data, a fuzzy model is achieved. The model can well simulate the nonlinear relationship among the driving frequency and rotary speed. Results of the experiments and measurements showed the validation of the fuzzy modeling. The paper shows that the nonlinear characteristics of USM are fit to be described. Furthermore, the presented method is general, and can be extended to other travelling wave type motors. To make precise control of USM, the proposed fuzzy model can be used. A fuzzy PID regulator is feasible to balance the variations in the operating conditions and preserve the desired rotary speed. As a future work, fuzzy logic will be combined to sliding mode technique to achieve a robust control scheme. The main advantage of fuzzy sliding mode control is that the control method achieves asymptotic stability of the closed-loop system.

\section{REFERENCES}

[1] T. Sashida and T. Kenjo, An introduction to ultrasonic motors, Oxford University Press, 1993.

[2] Hong Zhang, Hui Zhao, Weijia Shi and Yanlin Li, "A novel half-bridge drive circuit for ultrasonic motor based on FPGA," 2017 IEEE Transportation Electrification Conference and Expo, Asia-Pacific, Harbin, 2017, pp. 1-6.

[3] Hamed Mojallali, Rouzbeh Amini, Roozbeh Izadi Zamanabadi and Ali A. Jalali, "Systematic modeling for free stators of rotary piezoelectric ultrasonic motors," IEEE/ASME Transactions on Mechatronics, vol. 12, no. 2, pp. 219-223, 2007, DOI: 10.1109/TMECH.2007.892829

[4] Jian-Guo Zhang, Zhi-Li Long, Wen-Ju Ma, Guang-Hao Hu and Yang-Min Li, "Electromechanical dynamics model of ultrasonic transducer in ultrasonic machining based on equivalent circuit approach," Sensors 19, no. 6, pp. 1-6, 2019, DOI: $10.3390 / \mathrm{s} 19061405$

[5] Roland Ryndzionek, Łukasz Sienkiewicz, Michał Michna and Filip Kutt, "Design and experiments of a piezoelectric motor using three rotating mode actuators," Sensors 19, no. 23, pp. 1-16, 2019, DOI: $10.3390 / \mathrm{s} 19235184$

[6] Přemysl Janů, Josef Bajer, Pavel Dyčka and Radek Bystřický, "Precise experimental determination of electrical equivalent circuit parameters for ultrasonic piezoelectric ceramic transducers from their measured characteristics," Ultrasonics, vol. 112, 106341, 2021, DOI: 10.1016/j.ultras.2020.106341

[7] Dongsheng Zhang, Shiyu Wang and Jie Xiud, "Piezoelectric parametric effects on wave vibration and contact mechanics of traveling wave ultrasonic motor," Ultrasonics, vol. 81, pp. 118-126, 2017, DOI: 10.1016/j.ultras.2017.05.013

[8] J. Shi and W. Huang, "Improved DEA for motor's model identification," COMPEL - The International Journal for Computation and Mathematics in Electrical and Electronic Engineering, vol. 38 no. 6, pp. 1846-1854, 2019, DOI: 10.1108/COMPEL-05-2019-0185

[9] S. Jingzhuo and W. Huang, "Model reference adaptive iterative learning speed control for ultrasonic motor," in IEEE Access, vol. 8, pp. 181815-181824, 2020, DOI: 10.1109/ACCESS.2020.3029106

[10] S. Lu and S. Jingzhuo, "Nonlinear Hammerstein model of ultrasonic motor for position control using differential evolution algorithm," Ultrasonics, vol. 94, pp. 20-27, 2019, DOI: 10.1016/j.ultras.2018.12.012

[11] Jun Liu, Zi-Jie Niu, Hua Zhu and Chun-Sheng Zhao, "Design and experiment of a large-aperture hollow traveling wave ultrasonic motor with low speed and high torque," Applied Sciences, vol. 9, no. 19, pp. 1-16, sep 2019, DOI: 10.3390/app9193979 
[12] Hongpeng Yu, Qiquan Quan, Xinqi Tian and He Li, "Optimization and analysis of a u-shaped linear piezoelectric ultrasonic motor using longitudinal transducers," Sensors (Basel), vol. 18, no. 3, pp. 1-12, Mar. 2018, DOI: $10.3390 / \mathrm{s} 18030809$

[13] Dawei An, Weiqing Huang, Weiquan Liu, Jinrui Xiao, Xiaochu Liu and Zhongwei Liang, "Meshing drive mechanism of double traveling waves for rotary piezoelectric motors," Mathematics, vol. 9, no. 4, pp.1-19, 2021, DOI: $10.3390 /$ math9040445

[14] Xiang Li, Zhiwei Chen and Zhiyuan Yao, "Contact analysis and performance evaluation of standing-wave linear ultrasonic motors via a physics-based contact model," Smart Materials and Structures, vol. 28, no. 1, 2019, DOI: 10.1088/1361-665X/aaf11a

[15] Weijia Shi, Hui Zhao, Jie Ma and Yu Yao, "Dead-zone compensation of an ultrasonic motor using an adaptive dither," IEEE Transactions on Industrial Electronics, vol. 65, no. 5, pp. 3730-3739, 2018, DOI: 10.1109/TIE.2017.2760854

[16] T. Mashimo and K. Terashima, "Experimental verification of elliptical motion model in traveling wave ultrasonic motors," IEEE/ASME Transactions on Mechatronics, vol. 20, no. 6, pp. 2699-2707, 2015, DOI: 10.1109/TMECH.2015.2392126

[17] Shi Jingzhuo, Huang Wenwen and Zhou Ying, "T-S fuzzy control of travelling-wave ultrasonic motor," $J$ of Control Autom Electr Syst, vol. 31, pp. 319-328, 2020, DOI: 10.1007/s40313-019-00538-y

[18] Yunlai Shi, Jun Zhang, Yuyang Lin and Wenbo Wu, "Improvement of low-speed precision control of a butterflyshaped linear ultrasonic motor," IEEE Access, vol. 8, pp. 135131-135137, 2020, DOI: 10.1109/ACCESS.2020.3007773

[19] Jingwen Leng, Peng Pan, Zhike Xu, Tao Wang and Long Jin, "Design of low speed controller for traveling wave ultrasonic motor," 2019 22nd International Conference on Electrical Machines and Systems (ICEMS), Harbin, China, pp. 1-5, 2019, DOI: 10.1109/ICEMS.2019.8921667

[20] J. Wallaschek, "Piezoelectric ultrasonic motors," Journal of Intelligent Material Systems and Structures, vol. 6, pp. 71-83, 1995, DOI: 10.1016/B978-0-12-803581-8.03979-5

[21] Anis Maisarah Mohd Asry, Farahiyah Mustafa, Maizul Ishak, and Aznizam Ahmad, "Power generation by using piezoelectric transducer with bending mechanism support," International Journal of Power Electronics and Drive Systems (IJPEDS), vol. 10, no. 1, pp. 562-567, 2019, DOI: 10.11591/ijpeds.v10.i1.pp562-567

[22] A. Augustaitis and V. Jurènas, "Dynamics of trunk type robot with spherical piezoelectric actuators," International Journal of Robotics and Automation (IJRA), vol. 9, no. 2, pp. 113-122, 2020, DOI: 10.11591/ijra.v9i2.pp113-122

[23] Jianmin Qiu, Ying Yang, Xin Hong, Piotr Vasiljev, Dalius Mazeika and Sergejus Borodinas, "A disc-type high speed rotary ultrasonic motor with internal contact teeth," Appl. Sci. 11, no. 5, 2021, DOI: 10.3390/app11052386

[24] Yingxiang Liu, Shengjun Shi, Jipeng Yan, Weishan Chen and Dongmei Xu, "A novel piezoelectric actuator with two operating modes," Journal of Intelligent Material Systems and Structures, vol. 29, no. 6, pp. 1157-1164, 2018, DOI: $10.1177 / 1045389 X 17730921$

[25] N. W. Hagood and A. J. Mc Farland, "Modeling of a piezoelectric rotary ultrasonic motor," IEEE Transactions on Ultrasonics, Ferroelectrics, and Frequency Control, vol. 42, no. 2, pp. 210-224, 1995, DOI: 10.1109/58.365235

[26] He Li, Weishan Chen, Xinqi Tian and Junkao Liu, "An experiment study on temperature characteristics of a linear ultrasonic motor using longitudinal transducers," Ultrasonics, vol. 95, pp. 6-12, 2019, DOI: 10.1016/j.ultras.2019.03.003

[27] Lifeng Zhou, Zhiyuan Yao, Xiaoniu Li and Shichao Dai "Modeling and verification of thermal-mechanical-electric coupling dynamics of a V-shape linear ultrasonic motor," Sensors and Actuators A: Physical, vol. 298, 2019, DOI: 10.1016/j.sna.2019.111580

\section{BIOGRAPHIES OF AUTHORS}
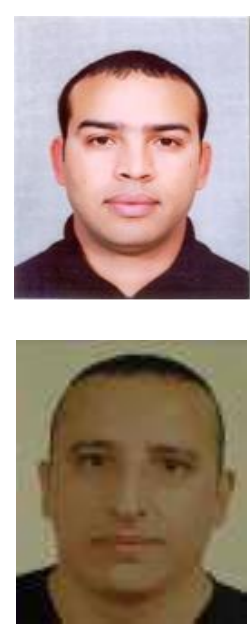

Youssef Baba was born in El Kelaa des Sraghna, Morocco, in 1982. He received the B.S. degree in Electrical Engineering from the National School of Applied Sciences of Marrakesh, Morocco, in 2007. He obtained the $\mathrm{PhD}$ degree in "Electrical Engineering" at Hassan 1 University, in 2018. He is currently an assistant professor at the department of Electrical Engineering, École Nationale Supérieure d'Arts et Métiers (ENSAM) Casablanca, Hassan II university, Morocco. His research interest includes motor control.

Mostafa Bouzi is a Professor in Electrical Engineering Department at Science and technology faculty, Hassan 1 University. He received the Engineering degree in Electrical Engineering, from Engineer school of Lille in France, obtaind the $\mathrm{PhD}$ degree in photovoltaic system control at university Hassan 1 and got his habilitation to supervise research at Hassan1 university. His current research activities are in the fields of electrical systems, renewable energy conversion systems and nonlinear control system. 